Karl T. Clebak, MD, MHA, FAAFP; Alexis Reedy-Cooper, MD, MPH; Michael T. Partin, MD; Christopher R. Davis, MD Penn State Health Milton S. Hershey Medical Center, Hershey (Drs. Clebak, Partin, and Davis); Penn State Health St. Joseph Medical Center, Reading (Dr. ReedyCooper).

Dkclebak@

pennstatehealth.psu.edu

The authors reported no potential conflict of interest relevant to this article.

doi: 10.12788/jfp.0198

\title{
A guide to the Tx of cellulitis and other soft-tissue infections
}

\section{Diagnostic and therapeutic priorities vary for the 8 types of infection reviewed.}

\section{PRACTICE RECOMMENDATIONS}

> Start trimethoprimsulfamethoxazole, clindamycin, doxycycline, minocycline, or a thirdor fourth-generation fluoroquinolone for patients with cellulitis likely caused by community acquired methicillinresistant Staphylococcus aureus (MRSA). A

> Consider culturing for MRSA and treating with oral doxycycline or trimethoprimsulfamethoxazole for resistant cases offolliculitis. (C)

> Perform complete surgical debridement promptly if necrotizing fasciitis is suspected. (c)

> Prescribe broad-spectrum antibiotics for necrotizing fasciitis, covering both anaerobes and aerobes including MRSA. (C)

Strength of recommendation (SOR)

A Good-quality patient-oriented evidence

B Inconsistent or limited-quality patient-oriented evidence

C Consensus, usual practice, opinion, disease-oriented evidence, case series kin and soft-tissue infections, frequently encountered in primary care, range from the uncomplicated erysipelas to the life-threatening necrotizing fasciitis. This review draws from the latest evidence and guidelines to help guide the care you provide to patients with cellulitis, orbital cellulitis, erysipelas, folliculitis, furuncles, carbuncles, abscesses, and necrotizing fasciitis.

\section{Cellulitis}

Cellulitis, an infection of the deep dermal and subcutaneous layers of the skin, has become increasingly common in recent years, with both incidence and hospitalization rates rising. ${ }^{1}$ Cellulitis occurs when pathogens enter the dermis through breaks in the skin barrier due to cutaneous fungal infections, trauma, pressure sores, venous stasis, or inflammation. The diagnosis is often made clinically based on characteristic skin findings-classically an acute, poorly demarcated area of erythema, warmth, swelling, and tenderness. Lymphangitic streaking and local lymphadenopathy may also be present. Infection often occurs on an extremity (although it can be found on other areas of the body) and is usually unilateral. Fever may or may not be present. ${ }^{2}$

I Likely responsible microorganisms. Staphylococcus aureus and Group A streptococci (often Streptococcus pyogenes) are common culprits. One systematic review that examined cultures taken of intact skin in cellulitis patients found S aureus to be about twice as common as $S$ pyogenes, with both bacteria accounting for a little more than $70 \%$ of cases. Of the remaining positive cultures, the most common organisms were alpha-hemolytic streptococcus, group B streptococcus, Pseudomonas aeruginosa, Clostridium perfringens, Escherichia coli, Pasteurella multocida, and Proteus mirabilis. ${ }^{3}$ Similarly, a systematic review of bacteremia in patients with cellulitis and erysipelas found that $S$ pyogenes, other beta-hemolytic strep, and $S$ aureus account for about $70 \%$ of cases (although $S$ aureus was responsible for just $14 \%$ ), with the remainder of cases 
caused by gram-negative organisms such as E coli and $P$ aeruginosa. ${ }^{4}$

- Treatment considerations. Strict treatment guidelines for cellulitis are lacking, but general consensus encourages the use of antibiotics and occasionally surgery. For mild and moderate cases of cellulitis, prescribe oral and parenteral antibiotics to cover for streptococci and methicillinsusceptible $S$ aureus, respectively. Expand coverage to include vancomycin if nasal colonization shows methicillin-resistant $S$ aureus (MRSA) or if you otherwise suspect prior MRSA exposure. Expanded coverage will also be needed if there is severe nonpurulent infection associated with penetrating trauma or a history of intravenous drug use, or the patient meets criteria for systemic inflammatory response syndrome. If patients are severely compromised (eg, neutropenic), it is reasonable to further add broad-spectrum coverage (eg, intravenous piperacillin-tazobactam or carbapenem). Typical duration of treatment is 5 to 7 days, although this should be extended if there is no clinical improvement.

Generally, cellulitis can be managed in the outpatient setting, although hospitalization is recommended if there are concerns for deep or necrotizing infection, if patients are nonadherent to therapy or are immunocompromised, or if outpatient therapy has failed. ${ }^{5}$ Furthermore, in an observational study of 606 adult patients, prior episodes of cellulitis, venous insufficiency, and immunosuppression were all independently associated with poorer clinical outcomes. ${ }^{2}$ Also treat underlying predisposing factors such as edema, obesity, eczema, venous insufficiency, and toe web abnormalities such as fissures, scaling, or maceration. ${ }^{5}$ Consider the use of prophylactic antibiotics for patients who have had 3 to 4 episodes of cellulitis despite attempts to treat predisposing conditions. Prophylactic antibiotic regimens include penicillin or erythromycin orally and penicillin G benzathine intramuscularly. ${ }^{5}$ Antibiotic regimens are summarized in the TABLE. ${ }^{5}$

\section{Orbital cellulitis}

Orbital cellulitis is an infection of the tissues posterior to the orbital septum. ${ }^{6,7}$ Periorbital, or preseptal, cellulitis occurs anterior to the orbital septum and is the more common of the 2 infections $-84 \%$ compared with $16 \%$ for orbital cellulitis. ${ }^{6}$ However, orbital cellulitis, which affects mainly children at a median age of 7 years, ${ }^{6}$ must be detected and treated early due to the potential for serious complications such as cavernous sinus thrombosis, meningitis, intracranial abscess, and vision loss. ${ }^{7}$ Chemosis (conjunctival edema) and diplopia are more commonly associated with orbital cellulitis and are seldom seen with preseptal cellulitis.

- Predominant causative organisms are $S$ pneumoniae, Moraxella catarrhalis, nontypeable Haemophilus influenzae, and group A streptococcus. The most common mechanism of infection is tracking from periorbital structures (eg, paranasal and ethmoid sinusitis). Other causes include orbital trauma/ fracture, periorbital surgery, and bacterial endocarditis. Clinically, patients present with limited ocular motility and proptosis associated with inflamed conjunctiva, orbital pain, headache, malaise, fever, eyelid edema, and possible decrease in visual acuity. The diagnosis is often made clinically and confirmed with orbital computed tomography (CT) with contrast, which can assist in ruling out intracranial involvement such as abscess.

Antibiotic therapy, generally administered intravenously, is recommended for at least 3 days or until orbital symptoms begin to resolve. Choose antibiotics effective against sinusitis-related pathogens (eg, S pneumoniae, H influenzae, M catarrhalis), $S$ aureus, and anaerobes. ${ }^{8}$ For instance, a regimen may include vancomycin for MRSA coverage, a third-generation cephalosporin, or metronidazole for anaerobic coverage if there is concern about intracranial involvement. Surgical intervention is often reserved for patients with inadequate response to antibiotic therapy, necessitating biopsy for pathogen identification, as well as drainage of large abscesses refractory to antibiotics.

\section{Erysipelas}

Erysipelas, a related yet distinct form of cellulitis, is a bacterial infection of the superficial

For orbital
cellulitis, choose
antibiotics
effective against
sinusitis-related
pathogens (eg,
$S$ pneumoniae,
H influenzae,
M catarrhalis),
S aureus, and
anaerobes.
anaerobes. 
When clinical exam alone is inconclusive when evaluating skin and softtissue infections in children and adolescents, consider using ultrasound to improve diagnostic accuracy.

\section{TABLE}

Antibiotic regimens for skin and soft-tissue infections ${ }^{5}$

\begin{tabular}{l|l}
\hline ANTIBIOTIC & ADULT DOSAGE \\
\hline Mild infection: Treat 5-7 days; extend if clinical improvement is insufficient \\
\hline Penicillin V & $250-500 \mathrm{mg}$ po q6h \\
\hline Amoxicillin & $500 \mathrm{mg}$ po tid, or $875 \mathrm{mg}$ po bid \\
\hline Cephalexin & $500 \mathrm{mg}$ po q6h \\
\hline Dicloxacillin & $500 \mathrm{mg}$ po q6h \\
\hline Ciprofloxacin & $500 \mathrm{mg}$ po bid \\
\hline Clindamycin* & 300 po qid, or $450 \mathrm{mg}$ po tid \\
\hline Doxycycline* & $100 \mathrm{mg}$ po bid \\
\hline Trimethoprim-sulfamethoxazole* & $1-2 \mathrm{DS}$ tablets po bid \\
\hline Minocycline* & $100 \mathrm{mg}$ po daily \\
\hline
\end{tabular}

Moderate systemic infection: Treat until there is clinical improvement and the patient is able to take oral antibiotics

\begin{tabular}{|c|c|}
\hline Penicillin G & 2-4 million units IV q4-6h \\
\hline Ceftriaxone & 1-2 g IV daily \\
\hline Vancomycin* & $15 \mathrm{mg} / \mathrm{kg}$ IV bid \\
\hline Linezolid* & $600 \mathrm{mg}$ po bid \\
\hline Daptomycin* & $4 \mathrm{mg} / \mathrm{kg}$ IV q24h \\
\hline Ceftaroline* & $600 \mathrm{mg}$ IV bid \\
\hline
\end{tabular}

Severe systemic infection: Treat until there is clinical improvement

\begin{tabular}{l|l}
\hline Vancomycin + piperacillin-tazobactam & $15 \mathrm{mg} / \mathrm{kg} \mathrm{IV} \mathrm{bid} \mathrm{+} 4.5 \mathrm{~g} \mathrm{IV} \mathrm{q8h}$ \\
\hline Vancomycin + imipenem-cilastatin & $\begin{array}{l}15 \mathrm{mg} / \mathrm{kg} \mathrm{IV} \mathrm{bid} \mathrm{+} 500 \mathrm{mg} \text { IV q6h or } 1 \mathrm{~g} \text { q8h IV for } \\
\text { fully susceptible organisms }\end{array}$ \\
\hline
\end{tabular}

Recurrent infections: Treat 4-52 weeks or as long as predisposing risk factors are present

\begin{tabular}{l|l}
\hline Penicillin $\mathrm{V}$ & $250-500 \mathrm{mg}$ po $\mathrm{q} 6 \mathrm{~h}$ \\
\hline Benzathine penicillin & 1.2 million units IM q2-4 weeks \\
\hline Erythromycin & $250 \mathrm{mg}$ po bid \\
\hline
\end{tabular}

* Coverage for suspected MRSA.

bid, twice a day; DS, double strength; IM, intramuscularly; IV, intravenously; MRSA, methicillin-resistant Staphylococcus aureus; po, by mouth; qid, 4 times a day; q6h, every 6 hours; tid, 3 times a day.

dermis and hypodermis and is commonly caused by group A streptococcus. ${ }^{5,9}$ Other less common organisms include $S$ aureus, $P$ aeruginosa, and enterobacteria. Erysipelas predominantly affects the lower extremities unilaterally $(\sim 90 \%)$; the arms and the face are the next most common locations. In addition to the rapid onset of well-demarcated erythema, pain, and swelling, patients may have fever and regional lymphadenopathy. Risk factors include portal of entry (eg, tinea pedis, ulceration), lymphedema, and diabetes. Complications of erysipelas include bullae from edema, abscess formation, and, rarely, bacteremia.

I Antibiotic treatment regimens include penicillin G, macrolides (reserved for those with penicillin allergies), fluoroquinolones, and cephalosporins, with duration of treatment ranging from 10 to 14 days depending on infection severity. Fever, pain, and erythema generally improve within 48 to 72 hours of antibiotic therapy. If there is no improvement, consider alternative diagnoses, such as necrotizing fasciitis. Recurrence rates following the initial episode of erysipelas are estimated at $10 \%$ of patients at 6 months and $30 \%$ at 3 years. ${ }^{10}$ 


\section{Folliculitis}

Inflammation of hair follicles is characterized by superficial inflammation with the development of perifollicular papules or pustules on an erythematous base. ${ }^{11,12}$ Folliculitis most commonly affects the face, scalp, thighs, buttocks, axillae, and inguinal areas. ${ }^{13}$ It may be caused by infection, an inflammatory reaction, or physical injury. Diagnosis is typically based on the patient's history and physical examination.

I Bacteria are the most common cause of infection, although fungi, viruses, and other entities can cause folliculitis. $S$ aureus (methicillin sensitive or methicillin resistant) is the most common pathogen; in the past, superficial pustular folliculitis attributed to $S$ aureus was referred to as Bockhart impetigo. Folliculitis secondary to $P$ aeruginosa, often seen after exposure to contaminated water or hot tubs, is frequently referred to as "hot tub folliculitis." Malassezia, a reported cause of fungal folliculitis, tends to occur in adolescents of either sex and men with high sebum production, is common in tropical climates, and can be associated with HIV or immunosuppression. . $^{11,12,14}$

Differential diagnosis of folliculitis includes pseudofolliculitis barbae, eosinophilic folliculitis, keratosis pilaris, acne vulgaris, candidiasis, contact dermatitis, impetigo, and miliaria. ${ }^{13}$ Pseudofolliculitis barbae is an inflammatory reaction to shaving, more commonly seen in darkly pigmented skin. Pseudofolliculitis develops when the hair shaft penetrates the wall of the follicle or directly enters the epidermis.

Initial treatment for mild disease includes the elimination of predisposing factors such as occlusion, moisture, and abrasion. The area should be kept clean and dry, avoiding friction. For localized disease, prescribe topical clindamycin, mupirocin ointment, or benzoyl peroxide. If symptoms fail to respond, prescribe a 7-day course of antibiotic that targets methicillin-sensitive $S$ aureus-eg, cephalexin or dicloxacillin. Also consider doxycycline, which has anti-inflammatory effects and is effective against MRSA. For refractory lesions, trimethoprim-sulfamethoxazole, clindamycin, or minocycline may be useful.
If you suspect pseudomonas, consider giving ciprofloxacin for 10 to 14 days for persistent lesions or if the patient is immunocompromised. ${ }^{13,15}$ Consider obtaining bacterial, fungal, or viral cultures for lesions that fail to respond to initial treatment.

\section{Furuncles/carbuncles/abscesses}

A furuncle, commonly referred to as a boil, is an infected hair follicle that becomes enclosed, creating a collection of pus. A carbuncle is a collection of furuncles that converge and drain through a single opening. An abscess is a localized collection of pus arising from within the dermis that can extend within deeper tissues. ${ }^{5}$ Furuncles, carbuncles, and abscesses are managed similarly with drainage and consideration for MRSA risk factors.

\section{I $S$ aureus is the most common cause} of these infections; $59 \%$ of skin abscesses are due to community-acquired MRSA. ${ }^{16}$ Anaerobes may contribute to the polymicrobial flora of skin abscesses. ${ }^{17}$ Risk factors for MRSA infection include a history of previous MRSA infection, diabetes, dialysis or renal failure, placement of an indwelling catheter or medical device, injection drug use, incarceration, close contact with a person with known MRSA infection or colonization, long-term care residence, hospitalization or surgery within the past 12 months, and high prevalence of MRSA in the community. ${ }^{5}$

I Ultrasound improves diagnostic accuracy. One study showed that when a clinical exam alone was inconclusive in evaluating skin and soft-tissue infections in children and adolescents, an ultrasound-assisted examination improved diagnostic accuracy. ${ }^{18}$ Sensitivity of the clinical examination was $43.7 \%$, compared with $77.6 \%$ for the clinical examination plus ultrasound..$^{18}$

Incision and drainage first. Ultrasound-guided needle aspiration, however, has not improved treatment efficacy compared with incision and drainage, ${ }^{19}$ the mainstay approach for abscesses. ${ }^{17}$ The procedure to drain a furuncle, carbuncle, or abscess should include the expression of all purulent material and the removal of all loculations if possible. Wound culture is recommended during incision and drainage per current guidelines. ${ }^{5}$

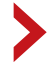

Do not routinely order cultures or prescribe antibiotics for uncomplicated abscesses. 


\section{$>$}

Monomicrobial infections, which account for $20 \%$ to $30 \%$ of cases of necrotizing fasciitis, are community acquired.
Simple dry dressings are convenient and effective, although some wounds may require packing. Tap water (that is potable) is suitable for wound cleansing. However, there is no strong evidence that irrigating wounds increases healing or reduces infection. ${ }^{20}$

I Routine use of antibiotics is not recommended for simple cutaneous abscesses. ${ }^{5,17,21}$ Evidence has been conflicting regarding empiric antibiotic coverage of MRSA following incision and drainage..$^{22-25}$ Guidelines recommend considering the initiation of antibiotics if there are multiple abscesses, gangrene, surrounding cellulitis, or systemic signs of infection, or if the host is immunocompromised. ${ }^{5}$

If MRSA is suspected, recommended antibiotic coverage includes trimethoprimsulfamethoxazole, clindamycin, doxycycline, or minocycline. ${ }^{5}$ If MRSA is identified, treatment options include dicloxacillin or cephalexin. For severe infections persisting after incision and drainage, in addition to oral antibiotic therapy, consider intravenous antibiotic options for MRSA: cefazolin, clindamycin, linezolid, nafcillin, telavancin, or vancomycin. ${ }^{5}$

\section{Necrotizing fasciitis}

Necrotizing fasciitis is a rare but potentially deadly infection of the skin and soft tissue. It progresses rapidly and spreads along fascial planes, leading to the necrosis of the superficial fascia. The infection often is more extensive than is indicated by superficial signs. Prompt diagnosis is imperative as necrotizing fasciitis is a surgical emergency. ${ }^{5,26}$ In the United States, 500 to 1500 cases of necrotizing fasciitis occur each year. ${ }^{27}$ Risk factors for necrotizing fasciitis include diabetes, peripheral vascular disease, malignancy, obesity, cirrhosis, renal failure, injection drug use, chronic corticosteroid therapy, alcohol abuse, malnutrition, and iatrogenic immunosuppression. $^{26,28}$

Necrotizing fasciitis may be polymicrobial or monomicrobial. Polymicrobial infection, also referred to as type I, is often due to multiple bacteria that originate from the bowel flora, typically including a mix of anaerobic and aerobic organisms. On average, there can be 5 infecting organisms identified per wound, although in some cases up to 15 organisms have been identified in a single wound..$^{5}$ Type I infection is often associated with tissue injury, abscess, or abdominal surgery. The majority of cases of necrotizing fasciitis are polymicrobial. ${ }^{27,28}$

Monomicrobial infection, also referred to as type II, is often due to group A streptococcus, $S$ aureus, vibrio spp, Aeromonas hyrophilio, or an anaerobic streptococci like peptostreptococcus spp. Typically monomicrobial infections, which account for $20 \%$ to $30 \%$ of cases of necrotizing fasciitis, are community acquired. 5,26,29,30

I Clinical presentation. In the early stages of disease, patients commonly complain of flu-like symptoms and extreme pain that is out of proportion to findings on the exam. Additional warning signs include fevers and other symptoms of toxicity such as tachycardia, hypotension, nausea, vomiting, and diarrhea. Later in the course, symptoms may localize to the affected area and include erythema, tense swelling, development of blisters or bullae, blackish blue discoloration of the skin, severe pain, and loss of sensation. In some cases involving gas-forming bacteria, tissue crepitus may be noted on exam. ${ }^{5,27-31}$

I Rely on clinical judgment to hasten surgical intervention. Laboratory or imaging findings may augment clinical judgment. But if you suspect necrotizing fasciitis, obtaining blood tests and imaging should not delay surgery. Blood tests that may aid in the diagnosis of necrotizing fasciitis include a complete blood count with differential; coagulation studies; a comprehensive metabolic panel; assays of lactate, C-reactive protein (CRP), and creatinine kinase; and blood cultures. Most often, patients with necrotizing fasciitis will have leukocytosis or leukopenia, evidence of hemolysis, thrombocytopenia, acute renal failure, and significantly elevated CRP.

On any imaging modality, indications of necrotizing fasciitis are inflammatory infiltration of the deep fascia on the affected side that is absent on the contralateral side, and the presence of subcutaneous air (which is a specific but rare finding). Imaging modalities may include CT or magnetic resonance imaging. A definitive diagnosis can only be made with surgical exploration of the involved area. 
Definitive microbiologic diagnosis will require culture of organisms from affected tissue or blood. 5,26,30,31

I First address any hemodynamic instability (hypotension is frequently encountered), followed by urgent surgical exploration, debridement of the wound, and antimicrobial therapy. Antibiotic treatment should align with probable pathogens and treatment should be continued until repeated surgical debridement is no longer necessary, clinical improvement is evident, and 48 to 72 hours have passed since defervescence.
A reasonable initial empiric regimen in adults would include an agent that is effective against group A streptococcus, gram-negative pathogens, and anaerobes, such as a carbapenem or a beta-lactam-beta-lactamase inhibitor such as piperacillin-tazobactam. Additionally, include an agent that targets MRSA, such as vancomycin, linezolid, or clindamycin. ${ }^{5}$

JFP

CORRESPONDENCE

Karl T. Clebak, MD, Department of Family and Community Medicine Residency Program, Penn State Health M.S. Hershey Medical Center, 500 University Drive, H154/C1613, Hershey, PA 17033; kclebak@pennstatehealth.psu.edu
References

1. Raff AB, Kroshinsky D. Cellulitis: a review. JAMA. 2016;316: 325-337.

2. Collazos J, de la Fuente B, García A, et al. Cellulitis in adult patients: a large, multicenter, observational, prospective study of 606 episodes and analysis of the factors related to the response to treatment. PLoS One. 2018;13: 0204036.

3. Chira S, Miller LG. Staphylococcus aureus is the most common identified cause of cellulitis: a systematic review. Epidemiol Infect. 2010;138:313-317.

4. Gunderson CG, Martinello RA. A systematic review of bacteremias in cellulitis and erysipelas. J Infect. 2012;64:148-155.

5. Stevens DL, Bisno AL, Chambers HF, et al. Practice guidelines for the diagnosis and management of skin and soft tissue infections: 2014 update by the Infectious Diseases Society of America. Clin Infect Dis. 2014;59:147-159.

6. Jain A, Rubin PA. Orbital cellulitis in children. Int Ophthalmol Clin. 2001;41:71-86.

7. Seltz LB, Smith J, Durairaj VD, et al. Microbiology and antibiotic management of orbital cellulitis. Pediatrics. 2011;127:e566-e572.

8. Nageswaran S, Woods CR, Benjamin DK, et al. Orbital cellulitis in children. Pediatr Infect Dis J. 2006;25:695-699.

9. Bonnetblanc J-M, Bédane C. Erysipelas. Am J Clin Dermatol. 2003;4:157-163.

10. Jorup-Rönström C, Britton S. Recurrent erysipelas: predisposing factors and costs of prophylaxis. Infection. 1987;15:105-106.

11. Clebak KT, Malone MA. Skin Infections. Prim Care. 2018;45: 433-454.

12. Luelmo-Aguilar J, Santandreu MS. Folliculitis: recognition and management. Am J Clin Dermatol. 2004;5:301-310.

13. Mengesha YM, Bennett ML. Pustular skin disorders: diagnosis and treatment. Am J Clin Dermatol. 2002;3:389-400.

14. Akaza N, Akamatsu H, Sasaki Y, et al. Malassezia folliculitis is caused by cutaneous resident Malassezia species. Med Mycol. 2009; 47:618-624.

15. Berger RS, Seifert MR. Whirlpool folliculitis: a review of its cause, treatment, and prevention. Cutis. 1990;45:97-98.

16. Fridkin SK, Hageman JC, Morrison M, et al. Methicillin-resistant Staphylococcus aureus disease in three communities. $N$ Engl J Med. 2005;352:1436-1444.

17. Meislin HW, Lerner SA, Graves MH, et al. Cutaneous abscesses anaerobic and aerobic bacteriology and outpatient management
Ann Intern Med. 1977;87:145-149.

18. Marin JR, Dean AJ, Bilker WB, et al. Emergency ultrasoundassisted examination of skin and soft tissue infections in the pediatric emergency department. Acad Emerg Med. 2013;20: 545-553.

19. Gaspari RJ, Resop D, Mendoza M, et al. A randomized controlled trial of incision and drainage versus ultrasonographically guided needle aspiration for skin abscesses and the effect of methicillin-resistant Staphylococcus aureus. Ann Emerg Med. 2011;57: 483-491.

20. Fernandez R, Griffiths R, Ussia C. Water for wound cleansing. Cochrane Database Syst Rev. 2002: CD003861.

21. Llera JL, Levy RC. Treatment of cutaneous abscess: a doubleblind clinical study. Ann Emerg Med. 1985;14:15-19.

22. Talan DA, Mower WR, Krishnadasan A, et al. Trimethoprimsulfamethoxazole versus placebo for uncomplicated skin abscess. NEngl J Med. 2016;374:823-832.

23. Korownyk C, Allan GM. Evidence-based approach to abscess management. Can Fam Physician. 2007;53:1680-1684.

24. Schmitz GR, Bruner D, Pitotti R, et al. Randomized controlled trial of trimethoprim-sulfamethoxazole for uncomplicated skin abscesses in patients at risk for community-associated methicillin-resistant Staphylococcus aureus infection. Ann Emerg Med. 2010;56:283-287.

25. Rajendran PM, Young D, Maurer T, et al. Randomized, double blind, placebo-controlled trial of cephalexin for treatment of uncomplicated skin abscesses in a population at risk for community-acquired methicillin-resistant Staphylococcus aureus infection. Antimicrob Agents Chemother. 2007;51:4044-4048.

26. Hunter J, Quarterman C, Waseem M, et al. Diagnosis and management of necrotizing fasciitis. Br J Hosp Med. 2011;72:391-395.

27. Hussein QA, Anaya DA. Necrotizing soft tissue infections. Crit Care Clin. 2013;29:795-806.

28. Puvanendran R, Huey JCM, Pasupathy S. Necrotizing fasciitis. Can Fam Physician. 2009;55:981-987.

29. Raven MC, Billings JC, Goldfrank LR, et al. Medicaid patients at high risk for frequent hospital admission: real-time identification and remediable risks. J Urban Health. 2009;86:230-241.

30. Ustin JS, Malangoni MA. Necrotizing soft-tissue infections: Crit Care Med. 2011;39:2156-2162.

31. Bystritsky R, Chambers H. Cellulitis and soft tissue infections. Ann Intern Med. 2018;168:ITC17- ITC32.
If you suspect necrotizing fasciitis, obtaining blood tests and imaging should not delay surgery.

\section{(\) Visit us @ mdedge.com/familymedicine}

